\title{
A CLASS OF C-TOTALLY REAL SUBMANIFOLDS OF SASAKIAN SPACE FORMS
}

\section{FILIP DEFEVER, ION MIHAI and LEOPOLD VERSTRAELEN}

(Received 25 November 1997; revised 28 April 1998)

Communicated by J. R. J. Groves

\begin{abstract}
Recently, Chen defined an invariant $\delta_{M}$ of a Riemannian manifold $M$. Sharp inequalities for this Riemannian invariant were obtained for submanifolds in real, complex and Sasakian space forms, in terms of their mean curvature. In the present paper, we investigate certain $C$-totally real submanifolds of a Sasakian space form $\tilde{M}^{2 m+1}(c)$ satisfying Chen's equality.
\end{abstract}

1991 Mathematics subject classification (Amer. Math. Soc.): 53C15, 53C40, 53C25.

\section{Introduction}

We consider $C$-totally real submanifolds $M^{n}$ of a Sasakian space form $\tilde{M}^{2 m+1}(c)$; let $H$ denote the mean curvature vector field of $M^{n}$ in $\tilde{M}^{2 m+1}(c)$. Precise definitions of the concepts used are given in Sections 2 and 3.

In [7] a general best possible inequality was obtained between the main intrinsic invariants of the submanifold $M^{n}$ on one side, namely its sectional curvature function $K$ and its scalar curvature function $\tau$, and its main extrinsic invariant on the other side, namely its mean curvature function $|H|$.

More precisely, in the Sasakian case, Chen's inequality, relating $K, \tau$ and $H$, reads:

$$
\inf K \geq \tau-\frac{n^{2}(n-2)}{2(n-1)}|H|^{2}-\frac{(n+1)(n-2)(c+3)}{8} .
$$

The first author is Postdoctoral Researcher N.F.W.O., Belgium; the second author is Research Fellow of the Research Council of the K.U. Leuven.

(C) 1998 Australian Mathematical Society 0263-6115/98\$A2.00+0.00 
[7] also classifies the $C$-totally real submanifolds $M^{n}$ of $\tilde{M}^{2 n+1}(c)$ with constant scalar curvature for which Chen's inequality becomes an equality.

In [6] a similar inequality for $\delta_{M}$ was established for totally real submanifolds of a complex space form; [6] and [4] contain also a classification of certain such submanifolds satisfying the equality.

In the present paper, we enlarge the investigation of [7] to the class of $C$-totally real submanifolds having nonconstant scalar curvature. Following [5], we consider $C$-totally real submanifolds $M^{n}$ in $\tilde{M}^{2 n+1}(c)$, satisfying Chen's equality, under some additional integrability condition. This extra condition then appropriately singles out, and conversely characterizes in some sense, a specific class of $C$-totally real submanifolds of $\tilde{M}^{2 n+1}(c)$ capturing the particular example with nonconstant scalar curvature, that fell outside the range of the classification result of [7]. This condition is stated in terms of some distribution, introduced in this context in [2]. More precisely, we prove the following theorem.

THEOREM 1. Let $\tilde{M}^{2 n+1}(c)$ be a Sasakian space form and $M^{n}$ an n-dimensional $(n>2) C$-totally real submanifold with nonconstant scalar curvature such that the subspaces

$$
\mathscr{D}(p)=\left\{X \in T_{p} M^{n} ; h(X, Y)=0, \forall Y \in T_{p} M^{n}\right\}, p \in M^{n},
$$

define a differentiable subbundle and its complementary orthogonal subbundle $\mathscr{D}^{\perp}$ is involutive. Then $M^{n}$ satisfies

$$
\delta_{M}=\tau-\inf K=\frac{(n-2)(n+1)(c+3)}{8},
$$

if and only if $M^{n}$ is locally congruent to an immersion

$$
\psi:\left(0, \frac{1}{2} \pi\right) \times_{\cos t} M^{2} \times_{\sin t} S^{n-3} \rightarrow S^{2 n+1}, \quad \psi(t, p, q)=(\cos t) p+(\sin t) q,
$$

where $M^{2}$ is a C-totally real minimal surface of $S^{5}$.

We remark that the example of a $C$-totally real submanifold with nonconstant scalar curvature satisfying Chen's equality given in [7] is included as a particular case of the above theorem, for $n=3$.

\section{C-totally real submanifolds of a Sasakian space form}

Let $\tilde{M}^{2 m+1}$ be an odd dimensional Riemannian manifold of class $C^{\infty}$ with Riemannian metric tensor field $g$. 
Let $\phi$ be a $(1,1)$-tensor field, $\xi$ a vector field, and $\eta$ a 1 -form on $\tilde{M}^{2 m+1}$, such that

$$
\begin{gathered}
\phi^{2} X=-X+\eta(X) \xi, \quad \phi \xi=0, \quad \eta(\phi X)=0, \quad \eta(\xi)=1, \\
g(\phi X, \phi Y)=g(X, Y)-\eta(X) \eta(Y), \quad \eta(X)=g(X, \xi) .
\end{gathered}
$$

If, in addition, $d \eta(X, Y)=g(\phi X, Y)$ for all vector fields $X, Y$ on $\tilde{M}^{2 m+1}$, then $\tilde{M}^{2 m+1}$ is said to have a contact metric structure $(\phi, \xi, \eta, g)$, and $\tilde{M}^{2 m+1}$ is called a contact metric manifold.

If moreover the structure is normal, that is if $[\phi X, \phi Y]+\phi^{2}[X, Y]-\phi[X, \phi Y]-$ $\phi[\phi X, Y]=-2 d \eta(X, Y) \xi$, then the contact metric structure is called a Sasakian structure (normal contact metric structure) and $\tilde{M}^{2 m+1}$ is called a Sasakian manifold. For more details and background, see the standard references $[1,10]$.

A plane section $\sigma$ in $T_{p} \tilde{M}^{2 m+1}$ of a Sasakian manifold $\tilde{M}^{2 m+1}$ is called a $\phi$-section if it is spanned by $X$ and $\phi X$, where $X$ is a unit tangent vector field orthogonal to $\xi$. The sectional curvature $\bar{K}(\sigma)$ with respect to a $\phi$-section $\sigma$ is called a $\phi$-sectional curvature. If a Sasakian manifold $\tilde{M}^{2 m+1}$ has constant $\phi$-sectional curvature $c, \tilde{M}^{2 m+1}$ is called a Sasakian space form and is denoted by $\tilde{M}^{2 m+1}(c)$.

The curvature tensor $\tilde{R}$ of a Sasakian space form $\tilde{M}^{2 m+1}(c)$ is given by [1]:

$$
\begin{aligned}
\tilde{R}(X, Y) Z= & \frac{c+3}{4}(g(Y, Z) X-g(X, Z) Y) \\
& +\frac{c-1}{4}(\eta(X) \eta(Z) Y-\eta(Y) \eta(Z) X+g(X, Z) \eta(Y) \xi-g(Y, Z) \eta(X) \xi \\
& +g(\phi Y, Z) \phi X-g(\phi X, Z) \phi Y-2 g(\phi X, Y) \phi Z),
\end{aligned}
$$

for any tangent vector fields $X, Y, Z$ to $\tilde{M}^{2 m+1}(c)$.

An $n$-dimensional submanifold $M^{n}$ of a Sasakian space form $\tilde{M}^{2 m+1}(c)$ is called a C-totally real submanifold of $\tilde{M}^{2 m+1}(c)$ if $\xi$ is a normal vector field on $M^{n}$. A direct consequence of this definition is that $\phi\left(T M^{n}\right) \subset T^{\perp} M^{n}$, which means that $M^{n}$ is an anti-invariant submanifold of $\tilde{M}^{2 m+1}(c)$, (hence their name of 'contact'-totally real submanifolds); see for example [9].

The Gauss equation implies that

$$
\begin{aligned}
R(X, Y, Z, W)= & \frac{1}{4}(c+3)(g(Y, Z) g(X, W)-g(X, Z) g(Y, W)) \\
& +g(h(X, W), h(Y, Z))-g(h(X, Z), h(Y, W)),
\end{aligned}
$$

for all vector fields $X, Y, Z, W$ tangent to $M^{n}$, where $h$ denotes the second fundamental form and $R$ the curvature tensor of $M^{n}$.

It is easily seen that

$$
2 \tau=n^{2}|H|^{2}-\|h\|^{2}+\frac{n(n-1)(c+3)}{4} .
$$




\section{Chen's inequality}

Let $M^{n}$ be an $n$-dimensional Riemannian manifold. Denote by $K(\pi)$ the sectional curvature of the plane section $\pi \subset T_{p} M^{n}, p \in M^{n}$. For any orthonormal basis $\left\{e_{1}, \ldots, e_{n}\right\}$ of the tangent space $T_{p} M^{n}$, the scalar curvature $\tau$ at $p$ is defined by

$$
\tau=\sum_{I \leq i<j \leq n} K\left(e_{i} \wedge e_{j}\right)
$$

For each point $p \in M^{\circ}$. uc put

$$
\operatorname{lnf} \mathcal{K}|| p \mid=\operatorname{Inf}\left\{K(\pi) ; \pi \subset T_{p} M^{n}, \operatorname{dim} \pi=2\right\} .
$$

The function inf $\Lambda^{\wedge}$ a a $u$ cll-defined function on $M^{n}$. Let $\delta_{M}$ denote the difference between the scalar curnslure and inf $K$, that is

$$
i_{v}(p)=\tau(p)-(\inf K)(p) ;
$$

$\delta_{M}$ is a well-defined Rienumnın invariant, which is trivial when $n=2$. The invariant $\delta_{M}$ was introduced $h$, (hen in [2], where he gave a sharp inequality for $\delta_{M}$ for submanifolds in resl pas $1 \mathrm{krm}$, and also obtained a classification of the minimal submanifolds wistsin: the cyuslity-case (see also [3]).

We now state the incyuslit, of Chen for the situation where the ambient space is a Sasakian space tırm: !

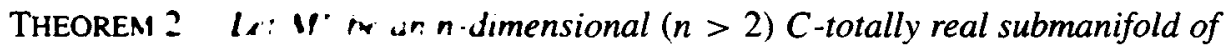

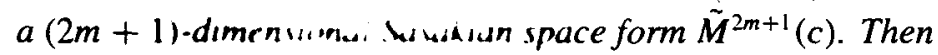

$$
\lambda_{v}: \frac{n}{2}=\left(\frac{n^{2}}{n-1}|H|^{2}+\frac{1}{4}(n+1)(c+3)\right) \text {. }
$$

Moreover, the equalis huld, at a point $p \in M^{n}$ if and only if there exist a tangent basis $\left\{e_{1}, \ldots, e_{n}\right\} \subset T_{r} M^{\prime}$ and a normal basis $\left\{e_{n+1}, \ldots, e_{2 m}, \xi\right\} \subset T_{p}^{\perp} M^{n}$ such that the shape operators take the following forms

$$
\begin{aligned}
A_{n+1} & =\left(\begin{array}{ccccc}
a & 0 & 0 & \ldots & 0 \\
0 & b & 0 & \ldots & 0 \\
0 & 0 & & \mu I_{n-2}
\end{array}\right), \quad a+b=\mu, \\
A_{r} & =\left(\begin{array}{ccccc}
h_{11}^{r} & h_{12}^{r} & 0 & \ldots & 0 \\
h_{12}^{r} & -h_{11}^{r} & 0 & \ldots & 0 \\
0 & 0 & & 0
\end{array}\right), \quad r \in\{n+2, \ldots, 2 m\},
\end{aligned}
$$

and $A_{\xi}=0$. 


\section{Submanifolds with maximal dimension}

We recall the following results, which we will need in the proof of Theorem 1.

PROPOSITION 3. Let $M^{n},(n>2)$, be a C-totally real submanifold of a Sasakian space form $M^{2 m+1}(c)$ which satisfies Chen's equality (4). Then for all $X$ tangent to $M^{n}, \phi X$ is perpendicular to $H$.

From now on, we restrict our attention to the totally real submanifolds $M^{n}$ of a Sasakian space form $\tilde{M}^{2 m+1}(c)$ with lowest possible codimension or equivalently with maximal dimension, that is, we assume that $m=n$.

In this case, under the assumptions of Proposition 3, it follows that the mean curvature vector field $H$ is in the direction of $\xi$ along $M^{n}$. Hence, we have the following corollary.

COROLLARY 4. Every $C$-totally real submanifold $M^{n}(n>2)$, of a Sasakian space form $M^{2 n+1}(c)$ which satisfies Chen's equality is minimal.

For a proof of these, as well as of the following Proposition 5, we refer to [7].

PROPOSITION 5. Let $M^{n}$ be an $n$-dimensional $(n>2)$ minimal $C$-totally real submanifold of a $(2 n+1)$-dimensional Sasakian space form $\tilde{M}^{2 n+1}(c)$. Then

$$
\delta_{M} \leq \frac{(n-2)(n+1)(c+3)}{8},
$$

and the equality holds at a point $p \in M^{n}$ if and only if there exists a tangent basis $\left\{e_{1}, \ldots, e_{n}\right\} \subset T_{p} M^{n}$ such that

$$
h\left(e_{1}, e_{1}\right)=\lambda \phi e_{1}, h\left(e_{1}, e_{2}\right)=-\lambda \phi e_{2}, h\left(e_{2}, e_{2}\right)=-\lambda \phi e_{1}, h\left(e_{i}, e_{j}\right)=0, i, j>2,
$$

where $\lambda \geq 0$ is given by

$$
4 \lambda^{2}=\frac{n(n-1)(c+3)}{4}-2 \tau
$$

Next, we prove Theorem 1. Before doing so, we remark that the conditions under which this theorem is stated, can be formulated in a slightly more explicit form. Indeed, let $M^{n}$ be a minimal $C$-totally real submanifold of a Sasakian space form $\tilde{M}^{2 n+1}(c)$. For each $p \in M^{n}$, we put

$$
\mathscr{D}(p)=\left\{X \in T_{p} M^{n} ; h(X, Y)=0, \forall Y \in T_{p} M^{n}\right\} .
$$


The geometric meaning of $\mathscr{D}$ is clear, namely $\mathscr{D}$ is the kernel of the second fundamental form $h$. In [2], it was shown that if $\operatorname{dim} \mathscr{D}(p)$ is constant, then it is completely integrable and its dimension is either $n$ or $n-2$.

In view of this last result, we can restate Theorem 1 in the following equivalent form, which is better suited for technical application.

THEOREM 2. Let $\tilde{M}^{2 n+1}(c)$ be a Sasakian space form and $M^{n}$ an $n$-dimensional $(n>2) C$-totally real submanifold with nonconstant scalar curvature such that:

(i) $\delta_{M}=\frac{1}{8}(n-2)(n+1)(c+3)$;

(ii) the distributions $\mathscr{D}$ and $\mathscr{D}^{\perp}$ are both completely integrable.

Then $M^{n}$ is, up to a homothety, locally congruent to an immersion

$$
\psi:\left(0, \frac{\pi}{2}\right) \times_{\cos t} M^{2} \times_{\sin t} S^{n-3} \rightarrow S^{2 n+1}, \quad \psi(t, p, q)=(\cos t) p+(\sin t) q,
$$

where $M^{2}$ is a $C$-totally real minimal surface of $S^{5}$.

PROOF. By Corollary 4, we know that $M^{n}$ is actually a minimal submanifold. Hence, by Proposition 5, there exists at every point $p \in M^{n}$ an orthonormal basis $\left\{e_{1}, \ldots, e_{n}\right\} \subset T_{p} M^{n}$ such that $h\left(e_{1}, e_{1}\right)=\lambda \phi e_{1}, h\left(e_{1}, e_{2}\right)=-\lambda \phi e_{2}, h\left(e_{2}, e_{2}\right)=-\lambda \phi e_{1}, h\left(e_{i}, e_{j}\right)=0, i, j>2$,

with $\lambda \neq 0$. We remark that in contrast to the situation studied in [7], $\lambda$ need not be a constant. Following the line of proof of the Lemmas 4.2 and 4.3 of [6], we can extend $\left\{e_{1}, \ldots, e_{n}\right\}$ to vector fields $\left\{E_{1}, \ldots, E_{n}\right\}$, which satisfy the above relations on a neighborhood of the point $p \in M^{n}$.

We observe that $M^{n}$ cannot be totally geodesic. Indeed, if $M^{n}$ were totally geodesic, (2) shows that in this case $M^{n}$ should in fact be a real space form. This would however imply that its scalar curvature is constant, which is excluded by assumption. Since we know from [2] that $\operatorname{dim} \mathscr{D}=n$ only occurs when $M^{n}$ is totally geodesic, we conclude that the dimension of $\mathscr{D}$ is $n-2$.

Hence, we may assume that locally, $\mathscr{D}^{\perp}$ is spanned by $\left\{E_{1}, E_{2}\right\}$. So, there exists $E_{3} \in \mathscr{D}$ (unique up to sign) such that

$$
\nabla_{E_{1}} E_{1}=b E_{2}+a E_{3},
$$

where $a, b$ are $C^{\infty}$-functions with $a \neq 0$.

We must have $c \neq-3$. Otherwise, $\tilde{M}^{2 n+1}$ is locally Euclidean and from the Gauss equation it follows that $R\left(E_{1}, E_{3}, E_{1}, E_{3}\right)=0$. This implies that $M^{n}$ is totally geodesic, which leads to a contradiction, as already remarked above. By a homothety, we can arrange that the ambient space has normalized $c=1$. 
We denote $\gamma_{i j}^{k}=g\left(\nabla_{E_{i}} E_{j}, E_{k}\right)$. Using the Codazzi equation, we find

$$
\gamma_{i j}^{1}=\gamma_{i j}^{2}=0, \quad \gamma_{11}^{i}=\gamma_{22}^{i}, \quad \gamma_{12}^{i}=\gamma_{21}^{i}=0, \quad \gamma_{i 1}^{2}=-\frac{1}{3} \gamma_{12}^{i} ; \quad i, j \geq 3 \text {. }
$$

Indeed, let $i, j \geq 3$. The equation $\left(\tilde{\nabla}_{E_{1}} h\right)\left(E_{i}, E_{j}\right)=\left(\tilde{\nabla}_{E_{i}} h\right)\left(E_{1}, E_{j}\right)$ is equivalent to $h\left(E_{1}, \nabla_{E_{i}} E_{j}\right)=0$, which implies $\gamma_{i j}^{1}=0$. Analogously, $\gamma_{i j}^{2}=0$.

Similarly, $\left(\tilde{\nabla}_{E_{2}} h\right)\left(E_{1}, E_{1}\right)=\left(\tilde{\nabla}_{E_{1}} h\right)\left(E_{2}, E_{1}\right)$ leads to $\gamma_{12}^{i}=-\gamma_{21}^{i}$. Since moreover $g^{-}$is involutive, we also have that $\gamma_{12}^{i}=\gamma_{21}^{i}$. Therefore $\gamma_{12}^{i}=0$. Finally, from $\left(\bar{\nabla}_{E} h\right)\left(E_{1}, E_{1}\right)=\left(\tilde{\nabla}_{E_{1}} h\right)\left(E_{i}, E_{1}\right)$ it follows that $E_{i}(\lambda)=\lambda \gamma_{11}^{i}$, which together with $\left(\tilde{\Gamma}_{\varepsilon} h\right)\left(E_{2}, E_{2}\right)=\left(\tilde{\nabla}_{E_{2}} h\right)\left(E_{i}, E_{2}\right)$ implies $\gamma_{22}^{i}=\gamma_{11}^{i}$.

In a similar way, we obtain

$$
E_{1}(\lambda)=-3 \lambda \gamma_{22}^{1}, \quad E_{2}(\lambda)=3 \lambda \gamma_{11}^{2} .
$$

Denoting $d=\gamma_{21}^{2}$, by the above relations we may write

$$
\nabla_{E_{2}} E_{1}=d E_{2}, \quad \nabla_{E_{2}} E_{2}=-d E_{1}+a E_{3}, \quad \nabla_{E_{i}} E_{1}=\nabla_{E_{i}} E_{2}=0 .
$$

Here. we clearly see that the assumption for the scalar curvature $\tau$ to be nonconstant w evential for the present proof. Indeed with $\tau$ constant, (2) shows that $\lambda$ would also the constant in this case. However, the above would then imply that $a=b=d=0$. But with $a=b=d=0$, the following final part of the proof is no longer applicable.

In order to finish the proof, it suffices to check Hiepko's condition from [8].

$W_{e}$ denote by $\mathscr{T}_{1}=\operatorname{span}\left\{E_{3}\right\}$ and $\mathscr{T}_{2}=\operatorname{span}\left\{E_{4}, \ldots, E_{n}\right\}$. So, it is sufficient to mone that:

(a) $\mathscr{T}_{1}$ is totally geodesic;

(h) $\bar{S}$ is spherical and $\mathscr{D}$ is totally geodesic;

(i) $\mathscr{Z}^{-}$is spherical and $\mathscr{T}_{1} \oplus \mathscr{D}^{\perp}$ is totally geodesic in $M^{n}$.

Indeed, we have $0=R\left(E_{1}, E_{3}, E_{i}, E_{1}\right)=-a \gamma_{33}^{i}, \forall i \geq 4$, which implies $\gamma_{33}^{i}=0$. So. we have that $\mathscr{T}_{1}$ is totally geodesic, thus proving (a).

Next. we prove (b). For $i, j \geq 4$

$$
\delta_{i j}=R\left(E_{i}, E_{1}, E_{j}, E_{1}\right)=g\left(\nabla_{E_{i}}\left(b E_{2}+a E_{3}\right)+\nabla_{\nabla_{E_{1}} E_{i}} E_{\mathrm{i}}, E_{j}\right)=a \gamma_{i 3}^{j} .
$$

Then $\nabla_{E_{i}} E_{j}=-(1 / a) \delta_{i j} E_{3}+Y, Y \in \mathscr{T}_{2} . \mathscr{T}_{2}$ is spherical if and only if $a$ is constant. But $E_{i}(a)=R\left(E_{i}, E_{1}, E_{3}, E_{1}\right)=0$.

For (c), obviously $\mathscr{T}_{1} \oplus \mathscr{D}^{\perp}$ is totally geodesic. It remains to show that $\mathscr{D}^{\perp}$ is spherical. Let $p, q \in\{1,2\}$; then $\nabla_{E_{p}} E_{q}=a \delta_{p q} E_{3}+Z, Z \in \mathscr{D}^{\perp}$. It follows that $\mathscr{D}^{\perp}$ is totally umbilical and its mean curvature vector $a E_{3}$ is parallel. Thus $\mathscr{D}^{\perp}$ is spherical. 
Using Hiepko's result [8], it follows that $M^{n}$ is locally isometric to a warped product manifold

$$
M^{n}=M_{0} \times_{\rho_{1}} M_{1} \times_{\rho_{2}} M_{2}
$$

Recall that by a result of [2], $\mathscr{D}$ is integrable and has dimension $n-2$. Since now both $\mathscr{T}_{1}$ and $\mathscr{D}$ are totally geodesic in $S^{2 n+1}$ and $\mathscr{T}_{2}$ is totally umbilical in $S^{2 n+1}, \operatorname{dim} M_{0}=1$ and $\operatorname{dim} M_{2}=n-3$. So, in fact, $M_{2}$ is locally a totally geodesic sphere of dimension $n-3: M_{2}=S^{n-3}$. Then, by counting dimensions, we see that $M_{1}$ being spherical is lying in $S^{5}$; since $M^{n}$ is minimal, $M_{1}$ is minimal in $S^{5}$ too. The warping functions can be determined from the equations (6), but we do not need explicit calculations. As the decomposition of $S^{2 n+1}$ into a warped product whose first factor is 1-dimensional is unique up to isometries (see [5]), following a similar argument as in [4], we can assume that

$$
\rho_{1}=\cos t, \quad \rho_{2}=\sin t
$$

Therefore, we obtain that $M^{n}$ is indeed immersed as desired.

\section{References}

[1] D. E. Blair, Contact manifolds in Riemannian geometry, Lecture Notes in Math. 509 (Springer, Berlin, 1976).

[2] B.-Y. Chen, 'Some pinching and classification theorems for minimal submanifolds', Arch. Math. (Basel) 60 (1993), 568-578.

[3] _- A Riemannian invariant for submanifolds in space forms and its applications (World Scientific, Singapore, 1994) pp. 58-81.

[4] B.-Y. Chen, F. Dillen, L. Verstraelen and L. Vrancken, "Totally real submanifolds of $\mathbf{C} P$ " satisfying a basic equality', Arch. Math. (Basel) 63 (1994), 553-564.

[5] _ 'Characterizing a class of totally real submanifolds of $S^{6}(1)$ by their sectional curvatures', Tôhoku Math. J. 47 (1995), 185-198.

[6] - 'An exotic totally real minimal immersion of $S^{3}$ in $\mathbf{C} P^{3}$ and its characterization', Proc. Roy. Soc. Edinburgh Sect. A 126 (1996), 153-165.

[7] F. Defever, I. Mihai and L. Verstraelen, 'B.-Y. Chen's inequality for C-totally real submanifolds of Sasakian space forms', Boll. Un. Mat. Ital. B 11 (1997), 365-374.

[8] S. Hiepko, 'Eine innere Kennzeichung der verzerrten Produkte', Math. Ann. 241 (1979), 209-215.

[9] K. Yano and M. Kon, Anti-invariant submanifolds, Lecture Notes in Pure and Appl. Math. (Marce] Dekker, New York, 1976).

[10] —, Structures on manifolds, Ser. Pure Math. 3 (World Scientific, Singapore, 1984). 
Zuivere en Toegepaste Differentiaalmeetkunde Departement Wiskunde

K. U. Leuven

Celestijnenlaan $200 \mathrm{~B}$

B - 3001 Leuven

Belgium

e-mail: filip.defever@wis.kuleuven.ac.be

Departement Wiskunde

K. U. Leuven

Celestijnenlaan 200 B

B - 3001 Leuven

Belgium,

and

Group of Exact Sciences

K.U. Brussel

Vrijheidslaan 17

B - 1081 Brussel

Belgium

e-mail: leopold.verstraelen@wis.kuleuven.ac.be
Faculty of Mathematics

University of Bucharest

Str. Academiei 14 70109 Bucharest

Romania

e-mail: imihai@math.math.unibuc.ro 